\title{
COMPARISON OF PERMANENT STAINING METHODS FOR THE LABORATORY DIAGNOSIS OF TRICHOMONIASIS
}

Camila Braz MENEZES(1), Mariana dos Santos MELLO(2) \& Tiana TASCA(1)

\begin{abstract}
SUMMARY
Trichomonas vaginalis is the etiologic agent of trichomoniasis, the most common non-viral sexually transmitted disease (STD) in the world. The diagnosis is based on wet mount preparation and direct microscopy on fixed and stained clinical specimens. The aim of this study was to compare the performance of different fixing and staining techniques used in the detection of T. vaginalis in urine. The smears were fixed and submitted to different methods of permanent staining and then, the morphological aspects of the parasites were analyzed and compared. The Papanicolaou staining with ethanol as the fixative solution showed to be the best method of permanent staining. Our data suggest that staining techniques in association with wet mount examination of fresh specimens contribute to increase the sensitivity in the diagnosis of trichomoniasis.
\end{abstract}

KEYWORDS: Trichomonas vaginalis; Diagnosis; Morphology; Fixing and staining techniques.

\section{INTRODUCTION}

Trichomoniasis, caused by the flagellate protozoan Trichomonas vaginalis, is the most common non-viral sexually transmitted disease (STD), responsible for 276 million new infections annually ${ }^{1}$. In Brazil, data depend on the region and the Health Ministry estimates a prevalence of $15 \%$. Trichomoniasis has been associated with serious health consequences for women, including adverse pregnancy outcomes, infertility, predisposition to cervical cancer, and pelvic inflammatory disease $^{3}$. Infection among men is a recognized cause of urethritis, and complications such as prostatitis, epididymitis, and infertility, including association with aggressive prostate cancers ${ }^{4}$. Moreover, trichomoniasis is a cofactor in human immunodeficiency virus (HIV) transmission and acquisition $^{5}$.

The most common method used in diagnosis of trichomoniasis is the wet mount examination, based on the parasite morphology and motility ${ }^{6}$. Despite being a method of easy implementation and low cost, it presents low sensitivity, since continued viability of parasites and well-trained microscopists are required. The parasite culture is not widespread used in clinical laboratories due to the time required for parasites growth and the costs associated with the culture medium ${ }^{7}$. The culture was considered the gold standard method and currently it was substituted by the nucleic acid amplification methods (NAATs) ${ }^{8}$. Molecular diagnosis such as polymerase chain reaction has increased the sensitivity and specificity in the detection of $T$. vaginalis $^{9}$, but this methodology is still developed only by few laboratories. Direct microscopy of fixed and stained clinical specimens remains one of the most used methods ${ }^{10-14}$, due to the easy implementation of stain techniques in the routine of many laboratories. Upon direct microscopy, urine and vaginal discharge are used for the identification of the parasites by observing their morphological characteristics. In addition to these clinical materials, $T$. vaginalis infection is frequently detected by Papanicolaou evaluation, during cervical cancer screening. A meta-analysis of articles, published between 1976 and 1998, on the ability of the Papanicolaou staining to detect vaginal trichomoniasis demonstrated $97 \%$ of specificity among cytologic evaluation. Hence, Papanicolaou staining could be a valuable tool in the diagnosis of this infection ${ }^{15}$.

Considering the high prevalence of trichomoniasis, the impact of the complications of this infection on public health systems, and the need to improve the diagnostic methods used in clinical laboratories, the aim of this study was to compare the performance of different fixing solutions and permanent staining techniques in diagnosis of $T$. vaginalis in culture medium and urine samples. The accurate identification of $T$. vaginalis trophozoites constitutes an important approach in the diagnosis of the infection.

\section{MATERIAL AND METHODS}

Samples: Trichomonas vaginalis isolate, 30236 (from the American Type Culture Collection) was cultured axenically in vitro on trypticaseyeast extract-maltose (TYM) medium, (pH 6.0), supplemented with $10 \%$ $(\mathrm{v} / \mathrm{v})$ heat-inactivated bovine serum, and incubated at $37^{\circ} \mathrm{C}( \pm 0.5)^{16}$. 
Urine samples were used as biological material (project with ethical approval by UFRGS Ethical Committee, number 18923).Two groups of smears were prepared: (i) trophozoites from the culture medium and (ii) urine samples experimentally infected with trophozoites (cell density 1.0 x $10^{5}$ trophozoites $/ \mathrm{mL}$ ) in order to reproduce the clinical diagnosis. Three independent experiments were performed (three independent cultures, $\mathrm{n}=3$ ), all in triplicate. From each triplicate, six slides were analyzed by two microscopists.

Staining procedures: Preparation of smears was performed using culture or urine that were deposited on the slide and flowed freely into vertical position to allow adequate physical attachment of the flagella and undulating membrane. Once prepared, the slides were dried at room temperature and submitted to different fixative and staining procedures as described below.

Fixative procedures: fixative solutions used in this study were methanol, ethanol, $2.0 \%$ paraformaldehyde (in PBS), and polyvinyl alcohol, that were chosen based on the literature review and in their usefulness in clinical laboratories ${ }^{12,17,18,19,20,21,22}$. The smears were submitted to staining methods for five minutes by immersion in fixative solutions once fixed.

Staining procedures: Distinct methods were evaluated: Giemsa and Gram, mostly used in clinical laboratories, and Papanicolaou, reference staining method in cytology laboratories ${ }^{11,12,18}$. After drying and staining, the smears were analyzed using optical microscopy under 1,000 x magnification.

Semi-quantitative analysis: Comparison between staining techniques was assessed by the evaluation of quantitative and qualitative aspects of the smears. Firstly, the quality of parasites fixation was evaluated based on the presence or absence of organisms by counting in 50 microscopic fields. The amount of parasites in 50 microscopic fields (quantitative analysis) was assessed as well as the main parasite morphological features - flagella, nucleus, axostyle, and undulating membrane (qualitative analysis). Trichomonas vaginalis pyriform morphology was also evaluated as criterion for the quality of fixation and staining. A semi-quantitative analysis was determined based on a score of percentage of parasites that exhibited the morphological criteria described, as follows: ++++ (one hundred percent of parasites exhibited the morphological criteria); +++ (seventy five percent of parasites exhibiting the morphological criteria); ++ (fifty percent of parasites exhibiting the morphological criteria); + (twenty-five percent of parasites exhibiting the morphological criteria). Data was analyzed independently by two microscopists.

\section{RESULTS}

In the present study, different fixative solutions and staining techniques were tested in combination, in order to evaluate the application of these methods in the diagnosis of trichomoniasis in clinical laboratories. Based on the morphological criteria applied, nucleus and flagella achieved the best scores (Table 1). On the other hand, in all combined conditions, both undulating membrane and axostyle did not stain.

Comparing the different methods used in this study, Giemsa staining provided nucleus as the only structure with clear visualization. However, a low number of parasites were detected in the smears, revealing the weak fixation of polyvinyl alcohol, ethanol and paraformaldehyde. In contrast, when the parasites were fixed with methanol as fixative solution, higher number of organisms were found with a well stained nucleus (Table 1). This finding reveals the importance of the appropriate choice of fixative solution for the use of Giemsa staining technique on routine $T$. vaginalis diagnosis. Gram staining was considered to present the poorest performance, since in two distinct combinations of fixative and trophozoite sources, parasites were not even fixated (Table 1). Moreover, when a few organisms were found to be fixed, the parasite's morphological characteristics were poorly visualized.

Papanicolaou staining showed the best performance among the techniques tested, since all parasites were stained. Considering culture as a sample, the staining showed the best results regardless of the fixative solution associated. Taking into account the urine samples with trophozoites, ethanol showed to be the best fixing solution, followed by methanol (Table 1). In view of morphological criteria adopted in this work, nucleus and flagella achieved the best scores within semiquantification. Furthermore, Papanicolaou staining was the only technique that evidenced the characteristic pyriform shape of T. vaginalis, especially in association with paraformaldehyde as fixing solution.

\section{DISCUSSION}

Our data demonstrates that Papanicolaou staining showed the best performance among those studied in this work, independently of the associated fixative solution applied.Nucleus and flagella were the morphological structures with better visualization on the stained smears. Undulating membrane and axostyle did not stain, failing to achieve the quantification criteria established in this work. Giemsa staining was not appropriated, since only nucleus was visualized in this technique. In the same way, Gram staining had the poorest performance in both organism fixation and morphological structures disclosure.

Surprisingly, Giemsa staining was not appropriated in elucidating $T$. vaginalis morphological aspects, as expected based on previous published data. Literature data indicate that results obtained on trichomonas staining are controversial. In a previous study, TASCA et al. $(2001)^{23}$ demonstrated superiority of Giemsa staining in comparison with iron hematoxylin staining in trophozoites of Tetratrichomonas didelphidis, similar to results from WENRICH et al. $(1944)^{24}$ for intestinal trichomonas also from culture. However, when cervicovaginal smears were analyzed, MASON et al. $(1976)^{20}$ showed best results in demonstrating morphological structures of T. vaginalis by Papanicolaou staining when comparing to Giemsa and acridine orange techniques.

Considering our results, Papanicolaou staining demonstrated adequate performance, and no differences were observed among all fixing solutions used. The combination of Papanicolaou staining with paraformaldehyde as fixing solution for trophozoites from in vitro culture was the only one capable to maintain the typical parasite pyriform shape. Commercially, the Papanicolaou staining in a cytological preservative is available with methanol and isopropanol, and our data showed acceptable performance of methanol and ethanol, although paraformaldehyde was better, revealing a new option for technique improvement. When urine was used as the biological sample, ethanol as fixing solution was the best combination with 
Table 1

Morphological characteristics of T. vaginalis observed in 50 fields, according to fixative solution/staining technique applied.

\begin{tabular}{|c|c|c|c|c|c|c|c|c|}
\hline \multirow{3}{*}{ MORPHOLOGICAL CHARACTERISTICS } & \multicolumn{8}{|c|}{ GIEMSA } \\
\hline & \multicolumn{8}{|c|}{ SAMPLE/FIXATIVE SOLUTION } \\
\hline & C/PVA & U/PVA & $\mathbf{C} / \mathrm{E}$ & $\mathbf{U} / \mathbf{E}$ & $\mathbf{C} / \mathbf{M}$ & $\mathbf{U} / \mathbf{M}$ & $\mathrm{C} / \mathrm{P}$ & $\mathbf{U} / \mathbf{P}$ \\
\hline Number of parasites & NF & NF & 43 & 7 & 60 & 2 & 9 & 3 \\
\hline Nucleus & $\mathrm{NF}$ & $\mathrm{NF}$ & + & ++++ & ++++ & ++++ & ++++ & - \\
\hline Flagela & NF & NF & - & - & - & - & - & - \\
\hline Undulating membrane & NF & NF & - & - & - & - & - & - \\
\hline Axostyle & NF & NF & - & - & - & - & - & - \\
\hline Shape & $\mathrm{NF}$ & $\mathrm{NF}$ & PA & PA & PA & PA & PA & PA \\
\hline
\end{tabular}

Shape

GRAM

\begin{tabular}{|c|c|c|c|c|c|c|c|c|}
\hline \multirow{2}{*}{ MORPHOLOGICAL CHARACTERISTICS } & \multicolumn{8}{|c|}{ SAMPLE/FIXATIVE SOLUTION } \\
\hline & C/PVA & U/PVA & $\mathbf{C} / \mathbf{E}$ & $\mathbf{U} / \mathbf{E}$ & $\mathbf{C} / \mathbf{M}$ & $\mathbf{U} / \mathbf{M}$ & $\mathbf{C} / \mathbf{P}$ & $\mathbf{U} / \mathbf{P}$ \\
\hline Number of parasites & 42 & 5 & $\mathrm{NF}$ & NF & NS & NS & 102 & 5 \\
\hline Nucleus & ++++ & ++++ & $\mathrm{NF}$ & NF & NS & NS & ++++ & ++++ \\
\hline Flagela & - & - & $\mathrm{NF}$ & NF & NS & NS & ++ & ++++ \\
\hline Undulating membrane & - & - & NF & NF & NS & NS & - & - \\
\hline Axostyle & - & - & $\mathrm{NF}$ & NF & NS & NS & - & - \\
\hline Shape & PA & PA & $\mathrm{NF}$ & $\mathrm{NF}$ & NS & NS & PA & PA \\
\hline
\end{tabular}

PAPANICOLAOU

MORPHOLOGICAL CHARACTERISTICS

SAMPLE/FIXATIVE SOLUTION

Number of parasites

Nucleus

Flagela

Undulating membrane

Axostile

Shape

PA PA

\begin{tabular}{cccccccc} 
C/PVA & U/PVA & C/E & U/E & C/M & U/M & $\mathbf{C} / \mathbf{P}$ & U/P \\
\hline 34 & 7 & 52 & 3 & 50 & 3 & 50 & 7 \\
++++ & - & ++++ & ++++ & ++++ & - & ++++ & - \\
++ & - & ++ & ++ & + & ++ & ++++ & - \\
- & - & - & - & - & - & - & - \\
- & - & - & - & - & - & - & - \\
PA & PA & PA & PA & PA & PA & PP & PA
\end{tabular}

C - Culture; U - Urine; PVA - Polyvinyl alcohol; E - Ethanol; M - Methanol; P - Paraformaldheyde; PA - predominantly ameboid; PP - predominantly piriform; NF - Not fixed; NS - Not stained; (-) Structure not observed.

Papanicolaou staining. It should be emphasized that parasite sources analyzed in this work were urine (as biological material) and culture, while in laboratory routine Papanicolaou staining is primarily used in cytological examinations. In these samples, T. vaginalis trophozoites present a greyish staining and are morphologically characterized with eccentric nucleus of pale appearance ${ }^{25}$. The flagella are rarely observed in cytological smears and parasite encircles cervical epithelial cells, which difficult the diagnosis decreasing sensitivity of the method. Based on our work, ethanol can be considered an appropriate fixing solution, but is important to highlight that paraformaldehyde was the only one that allowed the maintenance of the characteristic pyriform shape of the parasite, essential morphological characteristics for the identification of the parasite and correct diagnosis.
Thus, the association of staining techniques to the wet mount examination contributes to the successful diagnosis of trichomoniasis. Considering that Papanicolaou technique is already implemented in most laboratories and that urine is one of the most biological samples available, our report provides data for future studies to further define the positive/negative predictive values. Overall, we suggest that the association of wet mount examination with permanent staining techniques, mainly Papanicolaou, can improve the sensitivity in the diagnosis of trichomoniasis.

\section{ACKNOWLEDGMENTS}

This work was supported by the NANOBIOTEC-Brazil program from 
CAPES/Brazil (grant 23038.019022/2009-68) and CNPq/Brazil (grant 474930/2012-2). T.T. thanks CNPq for researcher fellowship

\section{REFERENCES}

1. World Health Organization. Global incidence and prevalence of selected curable sexually transmitted infections- 2008. Geneva: WHO; 2012.

2. Brasil. Ministério da Saúde. Secretaria de Vigilância em Saúde. Programa Nacional de DST e Aids. Prevalências e frequências relativas de doenças sexualmente transmissíveis (DST) em populações selecionadas de seis capitais brasileiras, 2005. Brasília: Ministério da Saúde/SVS/PNDST e AIDS; 2008.

3. Swygard H, Sena AC, Hobbs MM, Cohen MS. Trichomoniasis: clinical manifestations, diagnosis and management. Sex Trasm Infect. 2004;80:91-5.

4. Sutcliffe S, Neace C, Magnuson NS, Reeves R, Alderete JF. Trichomonosis, a common curable STI, and prostate carcinogenesis: a proposed molecular mechanism. PLoS Pathog. 2012;8:e1002801

5. Sorvillo F, Smith L, Kerndt P. Trichomonas vaginalis, HIV and African-Americans. Emerg Infect Dis. 2001;7:927-32.

6. Martin RD, Kaufman RH, Burns M. Trichomonas vaginalis vaginitis: a statistical evaluation of diagnostic methods. Am J Obstet Gynecol. 1963;87:1024-7.

7. Garber GE, Sibau L, Ma R, Proctor EM, Shaw CE, Bowie WR. Cell culture compared with broth for detection of Trichomonas vaginalis. J Clin Microbiol. 1987;25:1275-9.

8. Workowski KA, Bolan GA. Sexually transmitted diseases treatment guidelines, 2015. MMWR Recomm Rep. 2015; 64:1-137.

9. Riley DE, Roberts MC, Takayama T, Krieger JN. Development of a polymerase chain reaction based on diagnosis of Trichomonas vaginalis. J Clin Microbiol. 1992;30:46572.

10. Hughes HE, Gordon AM, Barr GT. A clinical and laboratory study of trichomoniasis of the female genital tract. J Obstet Gynecol Br Commonw. 1966;73:821-7.

11. Freeman F. A modified staining technique for Trichomonas vaginalis. S Afr Med J. 1958:32:1235.

12. Cree GE. Trichomonas vaginalis in Gram-stained smears. Br J Vener Dis.1968;44:226-7.

13. Fripp PJ, Mason PR, Super H. A method for the diagnosis of Trichomonas vaginalis using acridine orange. J Parasitol. 1975;61:966-7.
14. Lowe GH. A comparison of culture media for the isolation of Trichomonas vaginalis. Med Lab Technol. 1972;29:389-91.

15. Wiese W, Patel SR, Patel SC, Ohl CA, Estrada CA. A meta-analysis of the Papanicolaou smear and wet mount for the diagnosis of vaginal trichomoniasis. Am J Med. 2000;108:301-8.

16. Diamond LS. The establishment of various Trichomonas of animals and man in axenic cultures. J Parasitol. 1957;43:488-90.

17. Weinberger MW, Harger JH. Accuracy of the Papanicolaou smear in the diagnosis of asymptomatic infection with Trichomonas vaginalis. Obstet Gynecol. 1993;82:425-9.

18. Karaman U, Karadag N, Atambay M, Arserim Kaya NB, Daldal NU. A comparison of cytological and parasitological methods in the diagnosis of Trichomonas vaginalis. Turkiye Parazitol Derg. 2008;32:309-12.

19. Radonjic IV, Dzamic AM, Mitrovic SM, Arsic Arsenijevic VS, Popadic DM, Kranjcic Zec IF. Diagnosis of Trichomonas vaginalis infection: the sensitivities and specificities of microscopy, culture and PCR assay. Eur J Obstet Gynecol Reprod Biol. 2006;126:116-

20. Mason PR, Super H, Fripp PJ. Comparison of four techniques for the routine diagnosis of Trichomonas vaginalis infection. J Clin Pathol. 1976;29:154-7.

21. Folhadella IM, Fonseca AH, Jesus VLT, Pereira MJS. Desempenho de técnicas de coloração e fixação para o Tritrichomonas foetus (Riedmuller,1928) (Protozoa: Trichomonadida). Rev Bras Parasitol Vet. 2002;11:1-5.

22. EI Sayed Zaki M, Raafat D, El Emshaty W, Azab MS, Goda H. Correlation of Trichomona vaginalis to bacterial vaginosis: a laboratory-based study. J Infect Dev Ctries. 2010;4:156-63.

23. Tasca T, De Carli GA, Glock L, Jeckel Neto EA. Morphologic aspects of Tetratrichomona didelphidis isolated from opossums Didelphis marsupialis and Lutreolina crassicaudata. Mem Inst Oswaldo Cruz. 2001;96:265-71.

24. Wenrich DH. Morphology of the intestinal trichomonad flagellates in man and of simila forms in monkeys, cats, dogs, and rats. J Morphol. 1944;74:189-211.

25. Gompel C, Koss LG. Citologia das lesões inflamatórias. In: Gompel C, Koss LG, editores. Citologia ginecológica e suas bases anatomoclínicas. São Paulo: Editora Manole; 1997. p.61-71.

Received: 21 January 2015

Accepted: 17 April 2015 